\title{
DIRECT BOUNDARY ELEMENT METHOD FOR DYNAMICS IN A HALF-SPACE
}

\author{
By Paul S. NowaK and John F. HaLl
}

\begin{abstract}
An application of the direct boundary element method for solving the response of a linearly elastic half-space with a canyon cut into the surface is presented. This approach uses source solutions for an undamped half-space where the resulting singular integral equations are solved directly without adding any artificial damping. Solutions for the displacements on the canyon surface reveal an artificial resonance phenomenon when solving the exterior problem in the frequency domain. The use of an additional source loading in the boundary element method is shown to eliminate these resonances and yield accurate results. A method for solving the Rayleigh waves generated on the surface of the half-space caused by the canyon is shown.
\end{abstract}

\section{INTRODUCTION}

A problem that has attracted considerable attention in seismology is that of a wave propagating in a $2 \mathrm{D}$ half-space and incident onto a canyon cut into the surface (Sanchez-Sesma, 1987; Sanchez-Sesma et. al., 1985, 1982, 1979; Trifunac, 1973; Vogt et. al., 1988; Wong, 1979, 1982; Wong and Jennings, 1975; Wong and Trifunac, 1974). The solution approach employs a superposition procedure in the frequency domain; the difficult part requires solving for the motions caused by specified tractions on the surface of the canyon (without the incident wave). Described in the references are a variety of boundary solution techniques for this problem that appear to be adequate. Nevertheless, it is felt that a successful application of the traditional direct boundary element method to this exterior problem with a free surface would be of considerable practical interest, and such is described here.

Features of the presentation include:

- the use of source solutions obtained in a half-space rather than a full space, so the Raleigh pole is encountered and handled without adding damping;

- the use of linearly interpolated boundary elements with source loads applied at the nodes, so singularities are encountered and integrated, at nodes on the half-space surface and below;

- discussion of an artificial resonance phenomenon which can arise in solutions of exterior problems and the demonstration of a remedy;

- description of a procedure to compute the generated Rayleigh wave;

- accurate computation of an example problem which can be used to assess approximate solution methods.

\section{Statement of the Problem}

The domain of the posed problem is a linearly elastic, homogeneous, isotropic half-space with a canyon cut into the surface (Fig. 1). Specified tractions (tractions denoted by $T$, displacements by $u$, specified quantities with a bar) are applied to the surface of the canyon, and the remaining horizontal surface of the half-space is traction free. Neither the geometry nor the material properties nor 


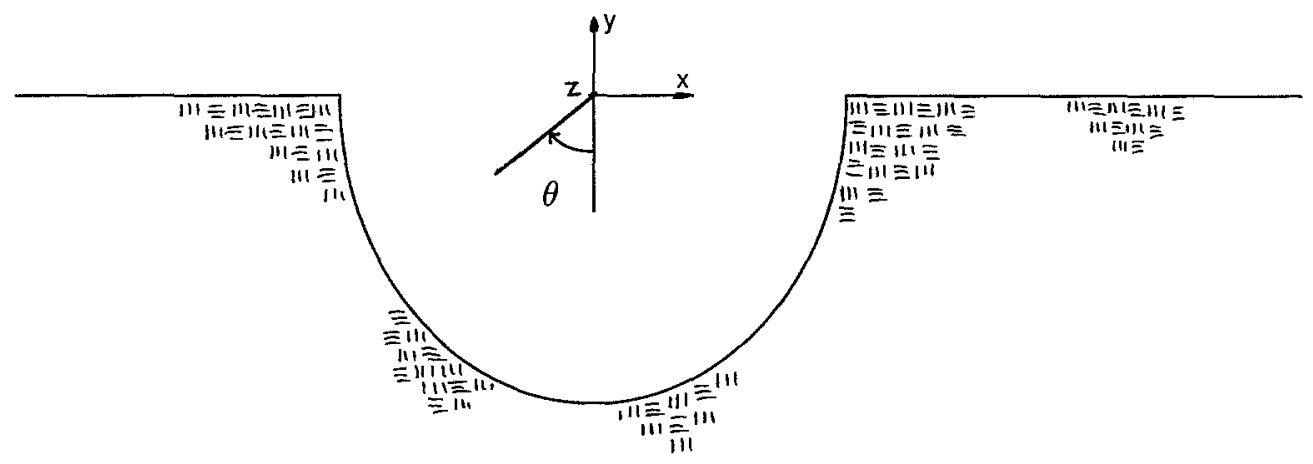

FIG. 1. Half-space with a canyon.

the applied tractions vary in the $z$ direction which is along the axis of the canyon. Thus, the problem uncouples into a pair of $2 \mathrm{D}$ ones: an antiplane shear problem involving $\bar{T}_{z}$ and $u_{z}$ and an in-plane problem (taken as a plane strain) involving $\bar{T}_{x}, \bar{T}_{y}, u_{x}$, and $u_{y}$.

Of interest are the transient responses $u_{x}(t), u_{y}(t)$, and $u_{z}(t)$ due to the time varying loads $\bar{T}_{x}(t), \bar{T}_{y}(t)$, and $\bar{T}_{z}(t)$. A frequency-domain approach is employed that involves solving the steady-state problem in which all quantities vary in time as $e^{-i \omega t}$, where $i=\sqrt{-1}$, for a range of frequencies $\omega$. In general, both the amplitudes of the responses $u_{x}(\omega), u_{y}(\omega)$, and $u_{z}(\omega)$ and loads $\bar{T}_{x}(\omega)$, $\bar{T}_{y}(\omega)$, and $\bar{T}_{z}(\omega)$ are complex valued and frequency dependent. For brevity, the $(\omega)$ notation will be dropped.

The tractions $\bar{T}_{z}$ in the antiplane shear problem generate $S H$ body waves that radiate away from the canyon. $P$ and $S V$ body waves are generated in the plane strain problem as well as Rayleigh waves which travel outward along the surface. Thus, the infiniteness of the domain must be represented in order to avoid wave reflections. Proper accounting of the Rayleigh waves in the plane strain problem adds considerable difficulty and computational effort.

\section{BASIC EQUATIONS}

The equations of motion (without body forces) and boundary conditions are

$$
\left.\begin{array}{c}
\mu\left(\frac{\partial^{2} u_{z}}{\partial x^{2}}+\frac{\partial^{2} u_{z}}{\partial y^{2}}\right)+\omega^{2} \rho u_{z}=0 \text { in } \Omega^{c} \\
T_{z}=\bar{T}_{z} \text { on } \Gamma \\
T_{z}=0 \text { on the horizontal surface } \\
\text { radiation condition at infinity }
\end{array}\right\}
$$

for antiplane shear and

$$
\left.\begin{array}{l}
(\lambda+\mu)\left(\frac{\partial^{2} u_{x}}{\partial x^{2}}+\frac{\partial^{2} u_{y}}{\partial x \partial y}\right)+\mu\left(\frac{\partial^{2} u_{x}}{\partial x^{2}}+\frac{\partial^{2} u_{x}}{\partial y^{2}}\right)+\omega^{2} \rho u_{x}=0 \\
(\lambda+\mu)\left(\frac{\partial^{2} u_{x}}{\partial x \partial y}+\frac{\partial^{2} u_{y}}{\partial y^{2}}\right)+\mu\left(\frac{\partial^{2} u_{y}}{\partial x^{2}}+\frac{\partial^{2} u_{y}}{\partial y^{2}}\right)+\omega^{2} \rho u_{y}=0
\end{array}\right) \text { in } \Omega^{e}
$$




$$
\left.\begin{array}{c}
T_{x}=\bar{T}_{x}, T_{y}=\bar{T}_{y} \text { on } \Gamma \\
T_{x}=T_{y}=0 \text { on the horizontal surface } \\
\text { radiation condition at infinity }
\end{array}\right\}
$$

for plane strain, where $\Omega^{c}=$ domain of half-space with canyon present, $\Gamma=$ surface of canyon, $\rho=$ density, $\omega=$ frequency (radians per second), $\mu=$ shear modulus, and $\lambda=$ Lamé constant.

To form the boundary integral equation, the weighted residual method and Green's theorem (twice) are applied to the equations of motion. The weighting functions $u_{x}^{*}, u_{y}^{*}$, and $u_{z}^{*}$ in the weighted residual method are chosen to satisfy

$$
\left.\begin{array}{c}
\mu\left(\frac{\partial^{2} u_{z}^{*}}{\partial x^{2}}+\frac{\partial^{2} u_{z}^{*}}{\partial y^{2}}\right)+\omega^{2} \rho u_{z}^{*}=\delta_{z}\left(x-x_{s}, y-y_{s}\right) \text { in } \Omega^{h} \\
T_{z}=0 \text { on the surface } \\
\text { radiation condition at infinity }
\end{array}\right\}
$$

for the antiplane shear problem, and

$$
\begin{aligned}
& (\lambda+\mu)\left(\frac{\partial^{2} u_{x}^{*}}{\partial x^{2}}+\frac{\partial^{2} u_{y}^{*}}{\partial x \partial y}\right)+\mu\left(\frac{\partial^{2} u_{x}^{*}}{\partial x^{2}}+\frac{\partial^{2} u_{x}^{*}}{\partial y^{2}}\right)+\omega^{2} \rho u_{x}^{*}=\delta_{x}\left(x-x_{s}, y-y_{s}\right) \\
& (\lambda+\mu)\left(\frac{\partial^{2} u_{x}^{*}}{\partial x \partial y}+\frac{\partial^{2} u_{y}^{*}}{\partial y^{2}}\right)+\mu\left(\frac{\partial^{2} u_{y}^{*}}{\partial x^{2}}+\frac{\partial^{2} u_{y}^{*}}{\partial y^{2}}\right)+\omega^{2} \rho u_{y}^{*}=0 \\
& \text { or }=0 \\
& =\delta_{y}\left(x-x_{s}, y-y_{s}\right) \\
& \text { in } \Omega^{h} \\
& \left.\begin{array}{c}
T_{x}=T_{y}=0 \text { on the surface } \\
\text { radiation condition at infinity }
\end{array}\right\}
\end{aligned}
$$

for the plane strain problem, where $\Omega^{h}$ is the half-space without the canyon and $\delta_{z}\left(x-x_{s}, y-y_{s}\right)$ is the Dirac delta function in the $z$ direction at point $\left(x_{s}, y_{s}\right)$, etc. for $\delta_{x}$ and $\delta_{y}$. The above weighting functions can be viewed as displacements due to the Dirac delta functions, which can be interpreted as line loads or sources. Restricting the point $\left(x_{s}, y_{s}\right)$ to be outside the domain $\Omega^{c}$ results in the following integral equations:

$$
\int_{\Gamma} T_{z}^{*} u_{z} d \Gamma=\int_{\Gamma} u_{z}^{*} \bar{T}_{z} d \Gamma
$$

for antiplane shear, and

$$
\int_{\Gamma}\left(T_{x}^{*} u_{x}+T_{y}^{*} u_{y}\right) d \Gamma=\int_{\Gamma}\left(u_{x}^{*} \bar{T}_{x}+u_{y}^{*} \bar{T}_{y}\right) d \Gamma
$$

for plane strain, where $u_{z}, u_{x}$, and $u_{y}$ are the unknown displacements and $\bar{T}_{z}$, $\bar{T}_{x}$, and $\bar{T}_{y}$ are the applied tractions at the canyon surface for the posed problem; $u_{z}^{*}$ and $T_{z}^{*}$ are the displacements and tractions at the (fictitious) canyon boundary from the solution of equations (5) and (6) in $\Omega^{h}$; and $u_{x}^{*}, u_{y}^{*}, T_{x}^{*}$, and $T_{y}^{*}$ are the displacements and tractions at the (fictitious) canyon boundary from 
the solution of equations (7) and (8) in $\Omega^{h}$. Note that because the source solutions satisfy the traction-free condition on the half-space surface, the domain of integration in equations (9) and (10) is only the surface of the canyon.

It is more convenient to place the source point $\left(x_{s}, y_{s}\right)$ on the boundary $\Gamma$ and, to avoid the singularity there, relocate the boundary inward and around the point (Fig. 2). Incorporating this boundary change in equations (9) and (10) and taking the limit as $r_{s} \rightarrow 0$ results in

$$
\begin{gathered}
\lim _{r_{\mathrm{s}} \rightarrow 0}\left(\int_{\Gamma-\Gamma^{\epsilon}} T_{z}^{*} u_{z} d \Gamma\right)+u_{z}\left(x_{s}, y_{s}\right) \lim _{r_{\mathrm{s}} \rightarrow 0}\left(\int_{\Gamma^{s}} T_{z}^{*} d \Gamma^{s}\right) \\
=\lim _{r_{s} \rightarrow 0}\left(\int_{\Gamma-\Gamma^{\epsilon}} u_{z}^{*} \bar{T}_{z} d \Gamma+\int_{\Gamma^{s}} u_{z}^{*} \bar{T}_{z} d \Gamma^{s}\right)
\end{gathered}
$$

and

$$
\begin{aligned}
& \lim _{r_{s} \rightarrow 0}\left(\int_{\Gamma-\Gamma^{\varepsilon}}\left(T_{x}^{*} u_{x}+T_{y}^{*} u_{y}\right) d \Gamma\right)+u_{x}\left(x_{s}, y_{s}\right) \lim _{r_{s} \rightarrow 0}\left(\int_{\Gamma^{s}} T_{x}^{*} d \Gamma^{s}\right) \\
& \quad+u_{y}\left(x_{s}, y_{s}\right) \lim _{r_{s} \rightarrow 0}\left(\int_{\Gamma^{s}} T_{y}^{*} d \Gamma^{s}\right) \\
& \quad=\lim _{r_{s} \rightarrow 0}\left(\int_{\Gamma-\Gamma^{e}}\left(u_{x}^{*} \bar{T}_{x}+u_{y}^{*} \bar{T}_{y}\right) d \Gamma+\int_{\Gamma^{s}}\left(u_{x}^{*} \bar{T}_{x}+u_{y}^{*} \bar{T}_{y}\right) d \Gamma^{s}\right) .
\end{aligned}
$$

The above formulations are merely statements of the Reciprocal Theorem and involve the solution of the posed problem in $\Omega^{c}$ as one set of loads and displacements, and the solution in $\Omega^{h}$ for the line sources as the other set of loads and displacements.

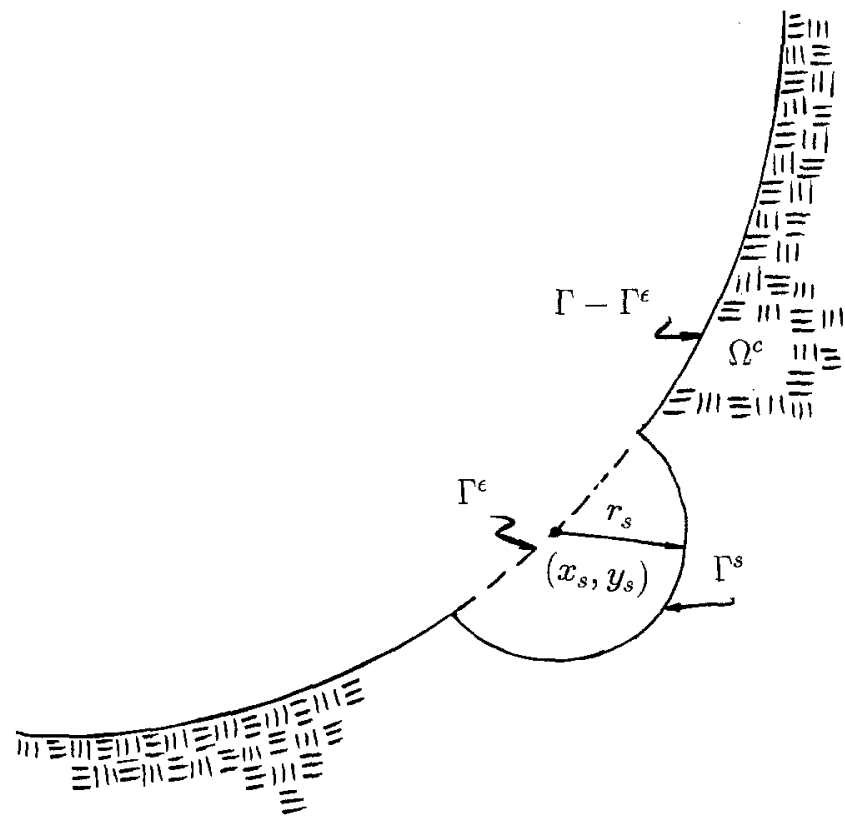

FIG. 2. Relocation of the canyon boundary around point $\left(x_{s}, y_{s}\right)$. 


\section{Solution of the Source Problem}

The solution of equations (5) and (6) for the displacements $u_{z}^{*}$ and tractions $T_{z}^{*}$ in the antiplane shear problem is obtained through the method of images. A line load $\delta_{z}\left(x-x_{s}, y-y_{s}\right)$ and its image $\delta_{z}\left(x-x_{s}, y+y_{s}\right)$ in an infinite fullspace are placed symmetrically about the plane $y=0$ at points $\left(x_{s}, y_{s}\right)$ and $\left(x_{s},-y_{s}\right)$. The displacements and stresses from these two line loads (Eringen and Suhubi; 1975) are then added together, which yields the surface $y=0$ (the fictitious surface of the half-space) traction free.

The method of images does not work completely in the plane strain case as only one of the two tractions on the fictitious half-space surface can be eliminated when superposing the image solution. Therefore, a superposition procedure is necessary: Case $\mathrm{A}$ in the full-space where the line load $\delta_{x}\left(x-x_{s}, y-y_{s}\right)$ or $\delta_{y}\left(x-x_{s}, y-y_{s}\right)$ and its image (actually the negative image) $\delta_{-x}\left(x-x_{s}, y\right.$ $\left.+y_{s}\right)$ or $\delta_{-y}\left(x-x_{s}, y+y_{s}\right)$ are applied so that $T_{y}^{A}(x, y=0)$ for $\delta_{x}, \delta_{-x}$ and $T_{x}^{A}(x, y=0)$ for $\delta_{y}, \delta_{-y}$ on the fictitious half-space surface become zero, and Case $\mathrm{B}$ in the half-space where the nonzero tractions $T_{x}^{A}(x, y=0)$ for $\delta_{x}, \delta_{-x}$ and $T_{y}^{A}(x, y=0)$ for $\delta_{y}, \delta_{-y}$ are removed from the surface, i.e., $T_{x}^{B}(x, y=0)=$ $\left.-T_{x}^{A}(x, y)=0\right)$ and $T_{y}^{B}(x, y=0)=0$ for $\delta_{x}, \delta_{-x}$ and $T_{y}^{B}(x, y=0)=$ $-T_{y}^{A}(x, y=0)$ and $T_{x}^{B}(x, y=0)=0$ for $\delta_{y}, \delta_{-y}$. Thus,

$$
g^{*}(x, y)=g^{A}(x, y)+g^{B}(x, y)
$$

where $g$ stands for $u_{x}, u_{y}, T_{x}$, or $T_{y}$.

The displacements and stresses from Case A are calculated from analytical expressions equation (3), whereas those of Case B are obtained through a spatial Fourier transform. The latter requires integration of the inverse Fourier transform

$$
g^{B}(x, y)=-\frac{K_{s}}{2 \pi} \int_{-\infty}^{\infty} h\left(\beta, y_{s}\right) g(\beta, y) \exp \left(i \beta K_{s}\left(x-x_{x}\right)\right) d \beta
$$

where $g^{B}(x, y)=$ either the displacement or stress response from Case $B$, $K_{s}=$ shear wave number $=\omega / C_{s}=1 / L_{s}, C_{s}=$ shear wave speed $=(\mu / \rho)^{1 / 2}$, $L_{s}=$ shear wavelength, $\beta=$ spatial Fourier transform parameter $=L_{s} /$ transform wavelength, $h\left(\beta, y_{s}\right) \cdot \exp \left(-i \beta_{s} K_{s} x_{s}\right)=$ spatial Fourier transform of $T_{x}^{A}(x, y=0)$ For $\delta_{x}, \delta_{-x}$ and $T_{y}^{A}(x, y=0)$ for $\delta_{y}, \delta_{-y}$, and $g(\beta, y)=$ transfer function for displacement or stress due to $T_{x}(x, y=0)=\exp \left(i \beta K_{s} x\right)$ for $\delta_{x}, \delta_{-x}$ and $T_{y}(x, y=0)=\exp \left(i \beta K_{s} x\right)$ for $\delta_{y}, \delta_{-y}$. The transfer function $g(\beta, y)$ can be expressed as

$$
g(\beta, y)=\frac{\hat{g}(\beta, y)}{\Re}
$$

where

$$
\Re=\left(2 \beta^{2}-1\right)^{2}-4 \beta^{2} \alpha \gamma,
$$

and $\alpha=\left(\beta^{2}-1\right)^{1 / 2}$ (negative root if $\beta<1$ and positive root if $\left.\beta \geqq 1\right), \gamma=\left(\beta^{2}\right.$ $\left.-\chi^{2}\right)^{1 / 2}$ (negative root if $\beta<\chi$ and positive root if $\beta \geqq \chi$ ), $\chi=C_{s} / C_{p}$ and $C_{p}=P$-wave speed $=((\lambda+2 \mu) / \rho)^{1 / 2}$. Expressions for $h\left(\beta, y_{s}\right)$ and $\hat{g}(\beta, y)$ are contained in Appendix 1.

The integration of the inverse Fourier transform is performed numerically noting that the integrand is either even or odd in the integration parameter $\beta$; 
therefore, only the $\beta \geqq 0$ portion need be considered (Appendix 2). Furthermore, the integrand is either real or imaginary for $\beta$ greater than $\beta_{s}$ (transform wavelength $=$ shear wavelength; note $\beta_{s}=1.0$ ). The upper limit of integration, $\beta_{\max }$, is chosen to produce a negligible truncation error and is selected to be that given by a transform wavelength equal to $1 / 6$ the sum of the depth of the source point and the depth of the response point, i.e.,

$$
\beta_{\max }=\frac{6.0 C_{s}}{\omega\left(y+y_{s}\right)} .
$$

The sampling increment $\Delta \beta$ of the integrand varies and is finer near $\beta_{p}$ (transform wavelength $=P$-wave wavelength; note $\beta_{p}=0.612$ for Poisson's ratio $(v)=0.20$ ), $\beta_{s}$, and $\beta_{r}$ (transform wavelength $=$ Rayleigh wavelength; note $\beta_{r}=1.0977$ for $v=0.20$ ) due to rapid variations of the integrand there. Up to $\beta^{\prime}=\beta_{r}+0.0081$, the sampling points (total of 256) are specified individually based on $v=0.20$; for $v \neq 0.20$, they are adjusted to fit the different values for $\beta_{p}$ and $\beta_{r}$. Beyond $\beta^{\prime}$, the spacing of the sampling points increases with $\beta$ as $\Delta \beta=\beta / 150$.

The actual integration is done in several parts. Between $\beta=0$ and the first sampling point below $\beta_{r}\left(\beta_{r}-\Delta\right.$, where $\left.\Delta=0.0001\right)$ and between $\beta_{r}+\Delta$ and $\beta^{\prime \prime}$ (where $\Delta \beta$ first exceeds $2 \pi / 10 K_{s}\left(x-x_{s}\right)$ ), the integrand is approximated as a quadratic across every $\Delta \beta$ pair using the three sampling points and integrated analytically. Between $\beta_{r}-\Delta$ and $\beta_{r}+\Delta$, care must be taken with the pole in the integrand; i.e., the denominator terms $\Re$ goes to zero at $\beta=\beta_{r}$. This portion of the integral is evaluated as the Cauchy principal value and includes the residue of the integrand at the pole. Approximating the numerator as linear between $\beta_{r}-\Delta$ and $\beta_{r}$ and between $\beta_{r}$ and $\beta_{r}+\Delta$ and linearizing $\mathfrak{R}$ about $\beta_{r}$ permits the Cauchy principal value to be obtained analytically, which is a sufficiently accurate procedure with the chosen value of $\Delta$. Results of the integration between $\beta_{r}-\Delta$ and $\beta_{r}+\Delta$ are included in the expressions in Appendix 2. The final part of the integration is between $\beta^{\prime \prime}$ and $\beta_{\max }$ where the $\cos \left(\beta K_{s}\left(x-x_{s}\right)\right)$ or $\sin \left(\beta K_{s}\left(x-x_{s}\right)\right.$ ) term (see Appendix 2) varies rapidly enough to make the quadratic approximation of the entire integrand inaccurate (more than $1 / 10$ of a wavelength in $\Delta \beta$, which defines $\beta^{\prime \prime}$ ). Therefore, the cos or sin term is separated out and integrated analytically with the rest of the integrand which is approximated as quadratic (again using the three sampling points over each $\Delta \beta$ pair).

\section{Solution of THE INTEGRAL EQUATION}

The displacements $u_{x}, u_{y}$, and $u_{z}$ in equations (11) and (12) are linearly interpolated within boundary elements (Fig. 3) from discrete values at the nodes, which become the unknowns of the problem (Banerjee and Butterfield, 1981). For the antiplane shear problem, algebraic equations in terms of the nodal displacements are generated by applying the line source $\delta_{z}$ to each node and performing the integrations in equation (11), resulting in $n$ equations for the $n$ unknowns, where $n$ is the number of nodes. For the plane strain problem, $2 n$ equations are generated for the $2 n$ unknowns by applying the line loads $\delta_{x}$ and $\delta_{y}$ to each node and performing the integrations in equation 12. Solution of the algebraic equations by matrix factorization yields the displacements $u_{x}, u_{y}$, and $u_{z}$ of the posed problem at the nodes. 


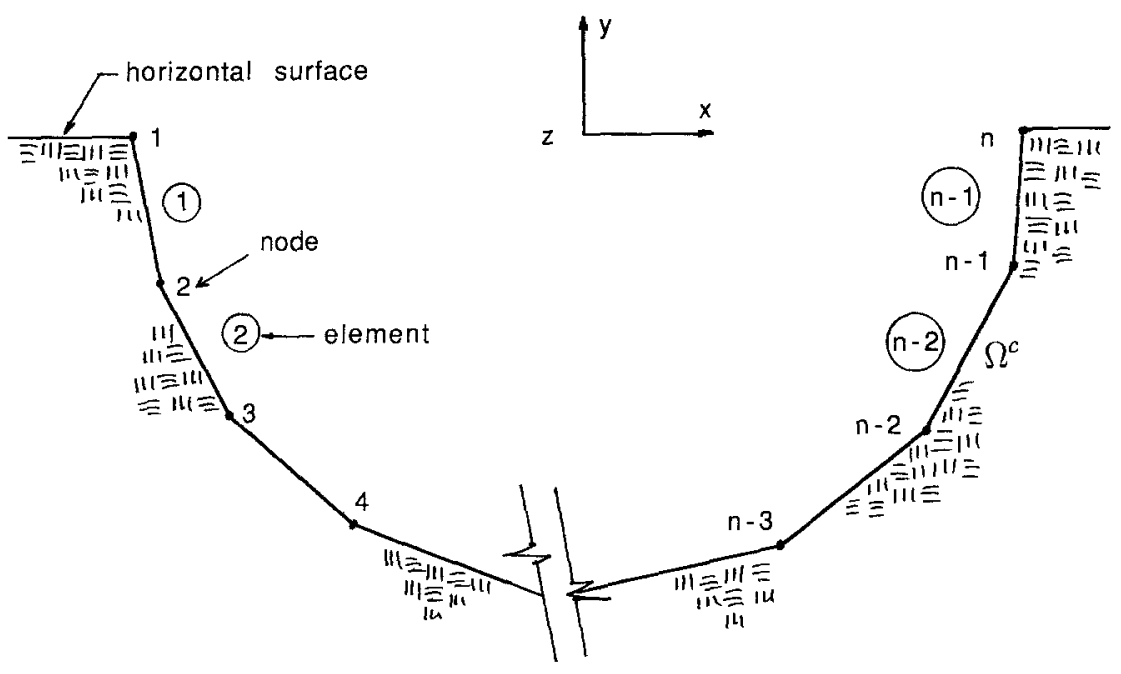

FIG. 3. Discretized canyon for the boundary element method.

For a line source applied at a particular node (node $s$ ), the integration in equations 11 and 12 over $\Gamma-\Gamma^{\epsilon}$ for all elements not adjacent to this node is performed by three-point Gauss quadrature. Within adjacent elements, where this integral must be evaluated in the limit as $r_{s} \rightarrow 0$, account must be taken of the singularities that occur in the line load displacements and tractions. Four procedures are needed depending on whether the integrand contains the $u_{k}^{*} \bar{T}_{k}$ term or the $T_{k}^{*} u_{k}$ term $k=x, y, x$, and whether node $s$ is at the top of the canyon on the horizontal free surface (nodes 1 and $n$, Fig. 3 ) or below the horizontal surface.

$u_{k}^{*} \bar{T}_{k}$, Node s Below Top of Canyon. The Hankel functions present in the line source displacements are expressed as the sum of $\operatorname{singular} \operatorname{terms}\left(\ln \left(r_{s}\right)\right.$ and, for the plane strain problem, $1 / r_{s}$ ) and a series of nonsingular terms (Abramowitz and Stegun, 1964). This produces $u_{k}^{*}=s t+n s$ where $s t=a$ constant times $\ln \left(r_{s}\right)$ (Appendix 3,1/r $r_{s}$ cancels) and $n s=$ nonsingular series; the singular term is independent of frequency $\omega$. The term $\left(s t \times \bar{T}_{k}\right)$ is integrated analytically after $\bar{T}_{k}$ is approximated as a quadratic using the three Gauss points as sampling points; the term $\left(n s \times \bar{T}_{k}\right)$ is integrated by three-point Gauss quadrature.

$u_{k}^{*} \bar{T}_{k}$, Node s at Top of Canyon. Closed form expressions for the displacements due to a line load at the surface of a half-space, such as in Eringen and Suhubi, 1975 for a buried line load, are available only for $\omega=0$. However, an integration scheme similar to that above can still be employed because singular term $s t$ is independent of frequency $\omega$ and, thus, known (constant times $\ln \left(r_{s}\right)$, Appendix 3 ) and because the nonsingular part $n s$ can be obtained at the Gauss points by $n s=u_{k}^{*}-s t$, where $u_{k}^{*}$ is computed by the inverse Fourier transform.

$T_{k}^{*} u_{k}$, Node $s$ Below Top of Canyon. Expansion of the Hankel functions in the lien load tractions along $\Gamma-\Gamma^{\epsilon}$ produces $T_{k}=s t+n s$, where $s t=$ constant times $1 / r_{s}$ (independent of $\omega$ ). However, for the antiplane shear problem, the constant of the singular term is zero along $\Gamma-\Gamma^{\epsilon}$; thus, no contribution to equation (11) from the term $T_{z}^{*} u_{z}$ results. Such is not the case in plane strain, where the terms to be integrated are $(s t+n s)\left(r_{s} / l\right)$ and $(s t+n s)\left(1-\left(r_{s} / l\right)\right)$ 
and where $l=$ length of the adjacent element and $u_{k}, k=x$ or $y$ has been expressed in terms of the linear interpolation functions. Except for st $\times 1$, all terms are integrable, and three-point Gauss quadrature is used. For st $\times 1$, however, the constants of the singular terms for the two adjacent elements are equal but of opposite sign, so no contribution results except at the end of the longer element, if the adjacent elements differ in length. No singularity occurs in this region, and analytical integration is used.

$T_{k}^{*} u_{k}$, Node $s$ at Top of Canyon. The line load traction $T_{z}^{*}$ is again zero, as in the case above. For plane strain, the $\omega=0$ solutions for $T_{z}^{*}$ and $T_{y}^{*}$ are also zero along $\Gamma-\Gamma^{\epsilon}$ except at $r_{s}=0$ where a delta function (line force) exists. Although no closed form solutions for $T_{z}^{*}$ and $T_{y}^{*}$ exist for $\omega>0$, it will be similar, but with an added continuous nonsingular variation along the adjacent element. Thus, noting that the line force at $r_{s}=0$ does not enter into the integrand along $\Gamma-\Gamma^{\epsilon}$, Gauss quadrature (three points) is used where $T_{x}^{*}$ and $T_{y}^{*}$ are computed at the Gauss points by the inverse Fourier transform.

The integration over $\Gamma^{s}$, also in the limit as $r_{s} \rightarrow 0$, is performed analytically using the expressions of Appendix 3 for $\Gamma^{s}$. Results for the terms on the left side of equations (11) and (12) are bounded and appear in appendix 4 . The limits of the $\Gamma^{s}$ integrals on the right side of equations (11) and (12) are zero because the line source displacements are singular only as $\ln \left(r_{s}\right)$.

\section{EXAMPLE SOLUTTON}

A computer implementation of the method described has been written and verified with the solutions contained in Wong, 1979, which include both antiplane shear and plane strain problems, as well as a number of other solutions. Details are presented in Nowak, 1988.

For an example problem, consider a semicircular canyon subjected to a pressure distribution $p=\left(2 p_{0} \mid \theta / \pi\right)$ (Fig. 4). The normalized horizontal displacement at the top of the canyon versus a normalized frequency is shown in Figure 5 as computed from uniform discretizations containing 16 and 32 elements. the resonances that occur are artificial because their width depends on the discretization. Frequencies of these resonances correspond to the natural frequencies of the material cut out of the half-space to form the canyon, fixed at the canyon boundary $\Gamma$ (Fig. 6). The source of the artificial resonances can be traced to a degeneracy in the boundary element matrix equation at these frequencies as described in Nowak, 1988 where the remedy was to use a fine enough discretization to localize the resonances and allow accurate interpolation of the true response. Another possibility is to move the line sources away

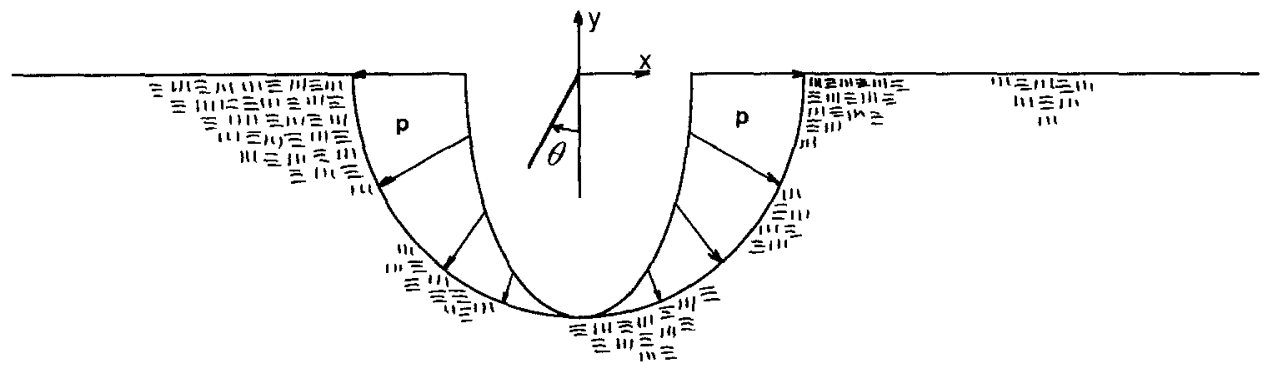

FIG. 4. Circular canyon with a pressure distribution, $p=\left(2 p_{0}|\theta|\right) / \pi$. 


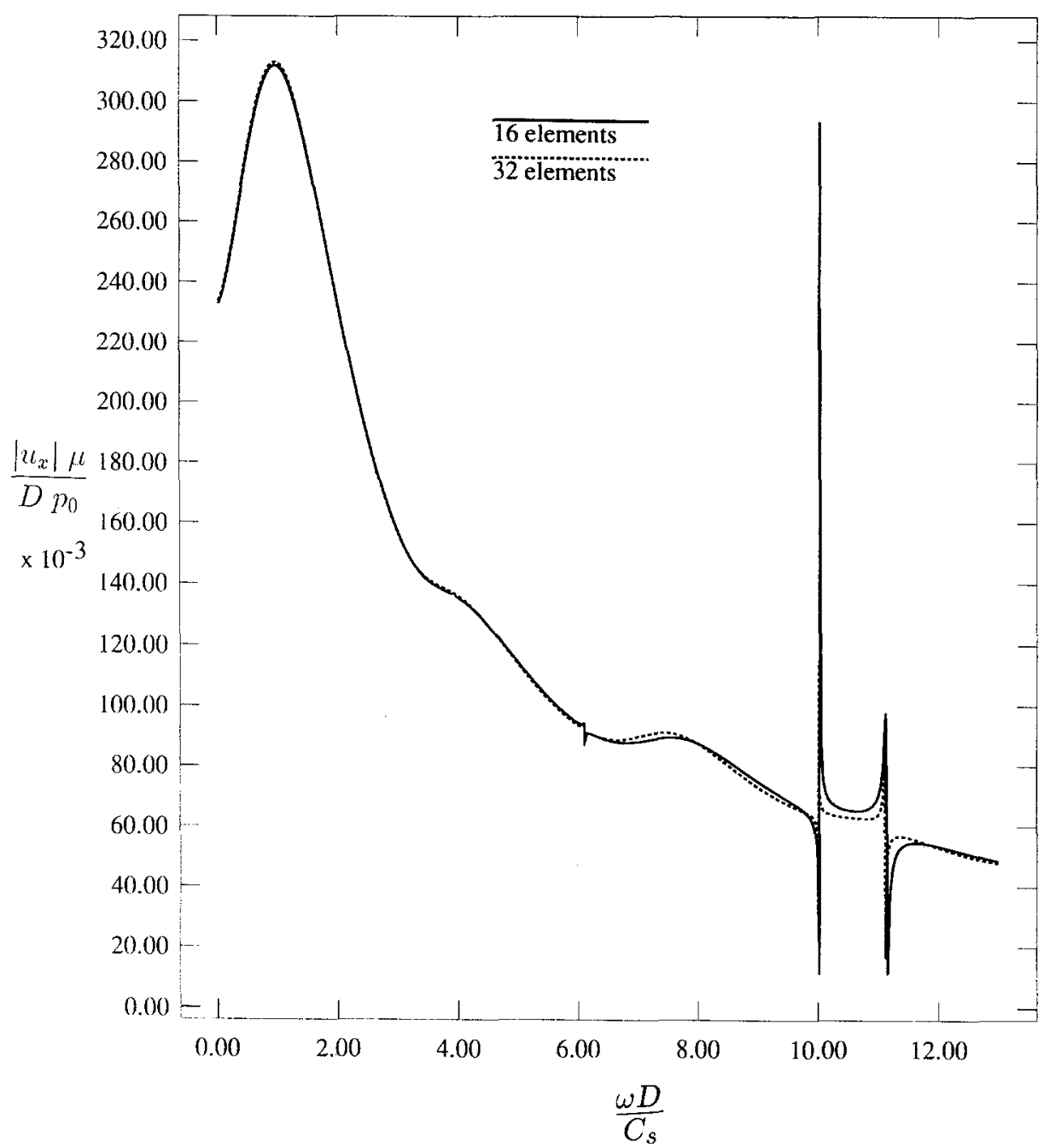

FiG. 5. Displacement response showing artificial resonances $(\nu=0.2)$.

from the (fictitious) boundary $\Gamma$, but this degrades accuracy and increases the complexity of the integrations in equations (11) and (12); the singularities resulting from placing the line sources on the (fictitious) boundary $\Gamma$ are relatively easy to handle.

The remedy to the artificial resonances employed here is to augment the line sources applied to the nodes with an additional source placed inside the (fictitious) canyon boundary which, when applied to the interior region (Fig. 6), would excite the modes where natural frequencies are the frequencies of the resonances to be eliminated. This extra source loading is placed far enough from the elements so that three-point Gauss quadrature can be used for the element integrations. For the symmetric pressure loading of the same problem, the extra source loading is chosen as symmetrical surface load $T_{y}(x, y)=2 \mathrm{~A} / D,-D / 4$ $\leqq x \leqq D / 4$, which is applied simultaneously with each line source. Results (Fig. 7) presented for $A=1.0$ show the effectiveness of the method. 


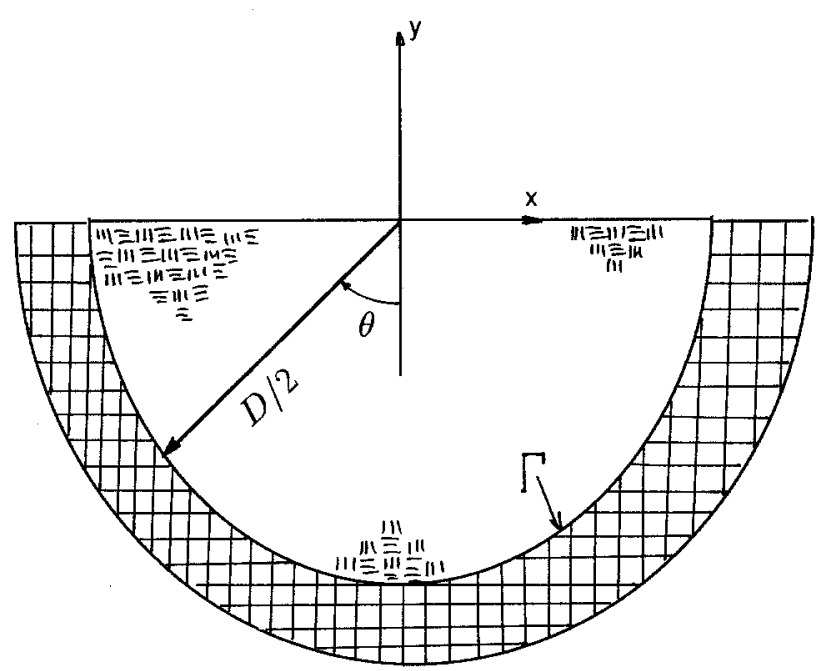

FIG. 6. Interior region fixed along canyon boundary.

Convergence for the example problem is demonstrated in Table 1 where the normalized $x$ and $y$ components of displacement at $\theta=0, \pi / 8, \pi / 4,3 \pi / 8$, and $\pi / 2$ on the canyon surface are presented for the 16 and 32 -element discretizations and a 64 element one at two frequencies. The results from the 64-element discretization can be considered highly accurate and can, therefore, be used to evaluate more approximate methods of solution.

\section{Generated Rayleigh Waves}

The amplitudes of the generated Rayleigh waves, one traveling in each direction outward from the canyon, can be determined by computing either the horizontal or vertical component of displacement (say, the vertical component) at one point on the surface to each side of the canyon and far from it $(x$ coordinate of such a point denoted by $x_{f}$ ). This requires that the line source $\delta_{y}$ be placed at this point which alters equation (10) to

$$
\int_{\Gamma}\left(T_{x}^{*} u_{x}+T_{y}^{*} u_{y}\right) d \Gamma+u_{y}\left(x_{f}, y=0\right)=\int_{\Gamma}\left(u_{x}^{*} \bar{T}_{x}+u_{y}^{*} \bar{T}_{y}\right) d \Gamma
$$

since the point $x, y=x_{f}, 0$ is contained in the domain $\Omega^{c}$. Equation (18) can be solved for the single unknown $u_{y}\left(x_{f}, y=0\right)$ after the boundary element solution of the posed problem for $u_{x}$ and $u_{y}$ at the canyon surface has been obtained. So, $T_{x}^{*}, T_{y}^{*}, u_{x}^{*}$, and $u_{y}^{*}$ in equation (18) represent the tractions and displacements at the fictitious canyon boundary in $\Omega^{h}$ due to the line source $\delta_{y}\left(x-x_{f}, y\right)$.

Responses $g^{*}(x, y)$ due to $\delta_{y}\left(x-x_{f}, y\right)$ in $\Omega^{h}$ can be obtained by integrating the inverse Fourier transform of equation (14) with $x_{f}$ replacing $x_{s}, h\left(\beta, y_{s}\right)=$ 1 , and $g(\beta, y)$ from equation (15) where the functions $\hat{g}(\beta, y)$ are defined by equations (A.8) to (A.12). Because $\left|x_{f}\right| \gg|x|$, the only contribution to the integral 


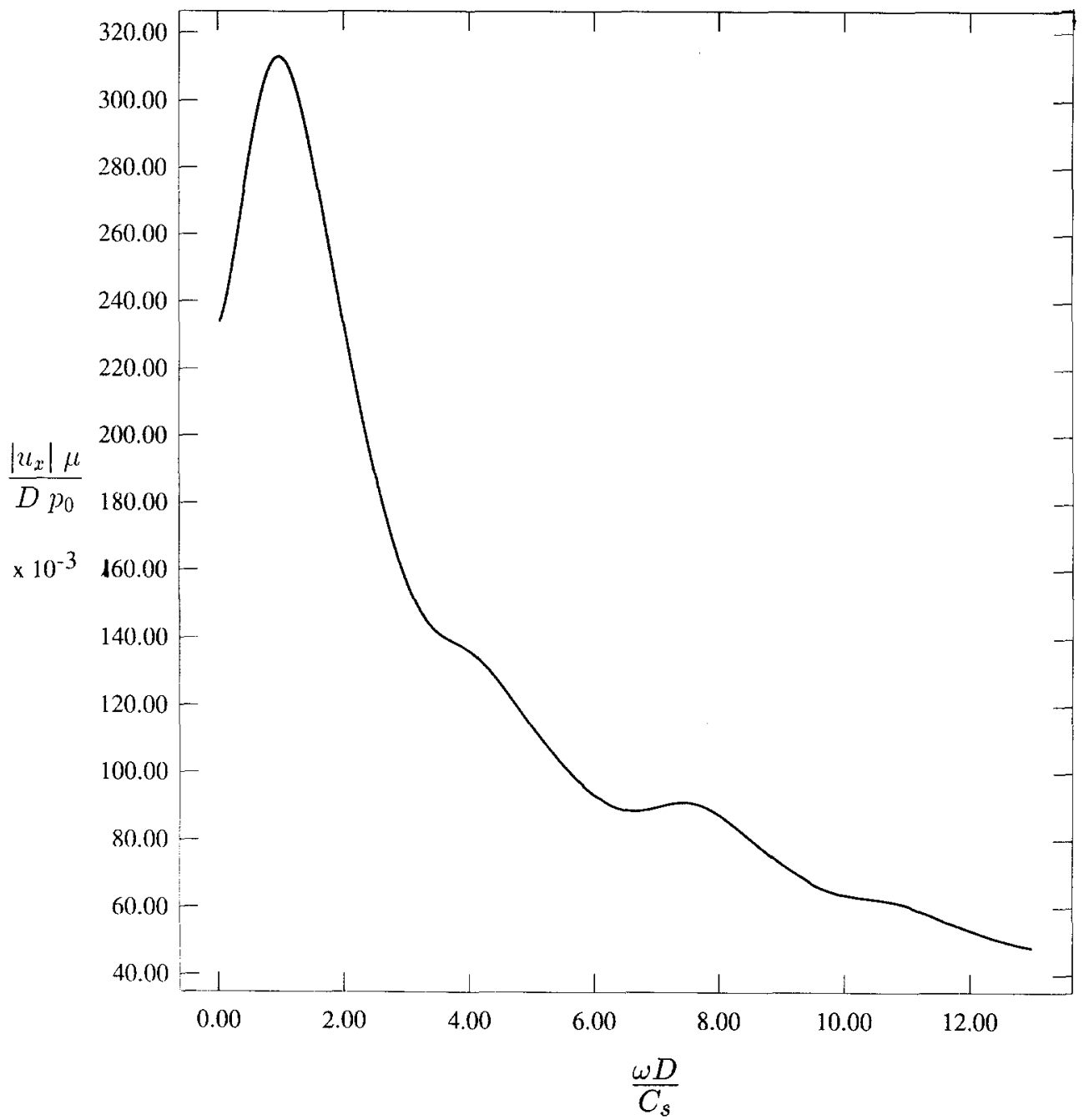

FIG. 7. Displacement response using the additional source $(\nu=0.2)$.

comes from the Rayleigh pole, and proper handling produces

$$
g^{*}(x, y)=\frac{K_{s} \hat{g}\left(\beta_{r}, y\right)}{R} \exp \left( \pm i \beta_{r} K_{s} x\right)
$$

which is merely the Rayleigh wave generated by $\delta_{y}\left(x-x_{f}, y\right)$ with + for $x_{f} \ll x$ (wave traveling in $+x$ direction) and - for $x_{f} \gg x$ (wave traveling in $-x$ direction). The expression in equation (19) applies to a particular set of coordinates $x_{f}$ which satisfy $\exp \left( \pm i \beta_{r} K_{s} x_{f}\right)=-i$.

\section{CONCLUSIONS}

The solution for surface displacements caused by tractions on a canyon cut into a $2 \mathrm{D}$ half-space is presented. These displacements are found by using the direct boundary element method based on source solutions for a half-space. This technique solves the most difficult part of the superposition problem for a wave 
TABLE 1

Displacements (REaL, Imaginary) on the Canyon Surface For Three Discretizations $\left(\times 10^{-2}\right)$

\begin{tabular}{|c|c|c|c|c|c|}
\hline $\mathbf{C}_{8}$ & $\theta$ & $\frac{\mathrm{u}_{0} \mu}{\mathrm{Dp}_{0}}$ & $\begin{array}{c}\text { 16-element } \\
\text { discretization }\end{array}$ & $\begin{array}{c}\text { 32-element } \\
\text { discretization }\end{array}$ & $\begin{array}{c}\text { 64-element } \\
\text { discretization }\end{array}$ \\
\hline 4 & $\frac{\pi}{2}$ & $\begin{array}{l}x \\
y\end{array}$ & $\begin{array}{c}(6.977,11.61) \\
(-3.177,2.762)\end{array}$ & $\begin{array}{c}(6.975,11.69) \\
(-3.124,2.683)\end{array}$ & $\begin{array}{c}(6.971,11.72) \\
(-3.100,2.655)\end{array}$ \\
\hline & $\frac{3 \pi}{8}$ & $\begin{array}{l}x \\
y\end{array}$ & $\begin{array}{l}(4.320,11.33) \\
(-.775,2.347)\end{array}$ & $\begin{array}{c}(4.311,11.37) \\
(-.818,2.229)\end{array}$ & \\
\hline & $\frac{\pi}{4}$ & $\begin{array}{l}x \\
y\end{array}$ & & & \\
\hline & $\frac{\pi}{8}$ & $\begin{array}{l}x \\
y\end{array}$ & $\begin{array}{l}(-.89 \\
(3.040\end{array}$ & $\begin{array}{l}3.888) \\
2.328)\end{array}$ & \\
\hline & 0 & $\begin{array}{l}x \\
y\end{array}$ & $\begin{array}{l}(0.000,0.000) \\
(2.561,2.536)\end{array}$ & & \\
\hline 9. & $\frac{\pi}{2}$ & $\begin{array}{l}x \\
y\end{array}$ & $\begin{array}{c}(1.556,7.330) \\
(-1.639,-.335)\end{array}$ & $\begin{array}{c}(1.506,7.147) \\
(-1.596,-.187)\end{array}$ & $\begin{array}{c}(1.495,7.071) \\
(-1.588,-.129)\end{array}$ \\
\hline & $\frac{3 \pi}{8}$ & $\begin{array}{l}x \\
y\end{array}$ & $\begin{array}{l}(0.281,5.177) \\
(1.137,0.617)\end{array}$ & $\begin{array}{l}(0.305,5.155) \\
(1.192,0.560)\end{array}$ & \\
\hline & $\frac{\pi}{4}$ & $\begin{array}{l}x \\
y\end{array}$ & & & \\
\hline & $\frac{\pi}{8}$ & $\begin{array}{l}x \\
y\end{array}$ & $\begin{array}{c}(-1.266,1.266) \\
(0.317,1.856)\end{array}$ & $\begin{array}{c}(-1.332,1.299) \\
(0.244,1.841)\end{array}$ & $\begin{array}{c}(-1.357,1.306) \\
(0.218,1.835)\end{array}$ \\
\hline & 0 & $\begin{array}{l}\mathrm{x} \\
\mathrm{y}\end{array}$ & $\begin{array}{c}(0.000,0.000) \\
(-.509,-.542)\end{array}$ & $\begin{array}{c}(0.000,0.000) \\
(-.338,-.491)\end{array}$ & $\begin{array}{c}(0.000,0.000) \\
(-.285,-.469)\end{array}$ \\
\hline
\end{tabular}

propagating in a half-space with a canyon. The description of a technique for solving for the Rayleigh waves that are generated by the canyon is also presented.

In solving the problem with the direct boundary element method, the resulting singular integral equations are solved directly without introducing any artificial damping. The solutions found through this technique reveal an artificial resonance phenomenon. By adding an additional source loading in the boundary element method this resonance can be eliminated. Results from an example problem, where tractions are applied to the surface of the canyon, show the effectiveness of this technique. These results are considered highly accurate and can be used to assess other approximate solution techniques.

\section{REFERENCES}

Abramowitz, M. and Stegun, I. A. (1964). Handbook of Mathematical Functions, Dover Publications, Inc., New York.

Banerjee, P. K. and Butterfield, R. (1981). Boundary Element Methods in Engineering Science, McGraw-Hill Book Company (UK).

Eringen, A. C. and Suhubi, E. S. (1975). Elastodynamics. Vol. 2, Academic Press, New York.

Nowak, P. S. (1988). Effect of Nonuniform Seismic Input on Arch Dams. Earthquake Engineering Research Laboratory Report NO. EERL 88-03, California Institute of Technology, Pasadena.

Sanchez-Sesma, F. J. (1987). Site effects on strong ground motion. Soil Dyn. Earthquake Eng. 6, 124-132. 
Sanchez-Sesma, F. J., Bravo, M. A. and Herrera, I. (1985). Surface Motion of Topographical Irregularities for Incident P, SV and Rayleigh Waves. Bull. Seism. Soc. Am. 75, 263-269.

Sanchez-Sesma, F. J., Herrera, I. and Aviles, J. (1982). A boundary method for elastic wave diffraction: application to scattering of SH waves by surface irregularities. Bull. Seism. Soc. Am., 72, 473-490.

Sanchez-Sesma, F. J. and Rosenbluth, E. (1979). Ground motion at canyons of arbitrary shape under incident SH waves. Int. J. Earthquake Eng. Struct. Dyn. 7, 441-450.

Timoshenko, S. P. and Goodier, J. N. (1970). Theory of Elasticity, 3rd Ed., McGraw-Hill Book Company, New York.

Trifunac, M. D. (1973). Scattering of plane SH waves by a semi-cylindrical canyon. Earthquake Eng. Struct. Dyn., 1, 267-281.

Vogt, R. F., Wolf, J. P., and Bachmann, H. (1988). Wave scattering by a canyon of arbitrary shape in a layered half-space. Int. J. Earthquake Eng. Struct. Dyn., 16, 803-812.

Wong, H. L. (1979). Diffraction of P, SV, and Rayleigh Waves by Surface Topographies. Report CE 79-05, University of Southern California, Los Angeles, California.

Wong, H. L. (1982). Effect of surface topography on the diffraction of P, SV and Rayleigh waves. Bull. Seism. Soc. Am., 72, 1167-1183.

Wong, H. L. and Jennings, P. C. (1975). Effect of Canyon Topography on strong ground motion. Bull. Seism. Soc. Am., 65, 5, 1239-1257.

Wong, H. L. and Trifunac, M. D. (1974). Scattering of plane SH waves by a semi-elliptical canyon. Int. J. Earthquake Eng. Struct. Dyn., 3, 157-169.

\section{APPENDIX 1. FunCTIONS $h\left(\beta, y_{s}\right)$ AND $\hat{g}(\beta, y)$}

Function $h\left(\beta, y_{s}\right)$ for $T_{x}^{A}(x, y=0)$

$$
h\left(\beta, y_{s}\right)=\left(2 \beta^{2}-1\right) \exp \left(y_{s} K_{s} \alpha\right)-2 \beta^{2} \exp \left(y_{s} K_{s} \gamma\right)
$$

Function $h\left(\beta, y_{s}\right)$ for $T_{y}^{A}(x, y=0)$

$$
h\left(\beta, y_{s}\right)=\left(2 \beta^{2}-1\right) \exp \left(y_{s} K_{s} \gamma\right)-2 \beta^{2} \exp \left(y_{s} K_{s} \alpha\right)
$$

Functions $\hat{g}(\beta, y)$ for $T_{x}(x, y=0)=\exp \left(i \beta K_{s} x\right)$

$$
\begin{gathered}
\hat{u}_{x}(\beta, y)=\frac{\alpha}{\mu K_{s}}\left[\left(2 \beta^{2}-1\right) \exp \left(y \alpha K_{s}\right)-2 \beta^{2} \exp \left(y \gamma K_{s}\right)\right] \\
\hat{u}_{y}(\beta, y)=\frac{i \beta}{\mu K_{s}}\left[2 \alpha \gamma \exp \left(y \gamma K_{s}\right)-\left(2 \beta^{2}-1\right) \exp \left(y \alpha K_{s}\right)\right] \\
\hat{\sigma}_{x x}(\beta, y)=2 i \beta \alpha\left[\left(2 \beta^{2}-1\right) \exp \left(y \alpha K_{s}\right)-\left(2 \gamma^{2}+1\right) \exp \left(y \gamma K_{s}\right)\right] \\
\hat{\sigma}_{x y}(\beta, y)=\left(2 \beta^{2}-1\right)^{2} \exp \left(y \alpha K_{s}\right)-4 \beta^{2} \alpha \gamma \exp \left(y \gamma K_{s}\right) \\
\hat{\sigma}_{y y}(\beta, y)=2 i \beta \alpha\left(2 \beta^{2}-1\right)\left[\exp \left(y \gamma K_{s}\right)-\exp \left(y \alpha K_{s}\right)\right]
\end{gathered}
$$

Functions $\hat{g}(\beta, y)$ for $T_{y}(x, y=0)=\exp \left(i \beta K_{s} x\right)$

$$
\begin{gathered}
\hat{u}_{x}(\beta, y)=\frac{i \beta}{\mu K_{s}}\left[\left(2 \beta^{2}-1\right) \exp \left(y \gamma K_{s}\right)-2 \alpha \gamma \exp \left(y \alpha K_{s}\right)\right] \\
\hat{u}_{y}(\beta, y)=\frac{\gamma}{\mu K_{s}}\left[\left(2 \beta^{2}-1\right) \exp \left(y \gamma K_{s}\right)-2 \beta^{2} \exp \left(y \alpha K_{s}\right)\right] \\
\hat{\sigma}_{x x}(\beta, y)=4 \beta^{2} \alpha \gamma \exp \left(y \alpha K_{s}\right)-\left(2 \beta^{2}-1\right)\left(2 \gamma^{2}+1\right) \exp \left(y \gamma K_{s}\right) \\
\hat{\sigma}_{x y}(\beta, y)=2 i \beta \gamma\left(2 \beta^{2}-1\right)\left[\exp \left(y \gamma K_{s}\right)-\exp \left(y \alpha K_{s}\right)\right] \\
\hat{\sigma}_{y y}(\beta, y)=\left(2 \beta^{2}-1\right)^{2} \exp \left(y \gamma K_{s}\right)-4 \beta^{2} \alpha \gamma \exp \left(y \alpha K_{s}\right)
\end{gathered}
$$


APPENDIX 2. INTEGRATION OF THE INVERSE FOURIER TRANSFORM With symmetric transfer function $g(\beta, y)$

$$
\begin{aligned}
g^{B}(x, y)= & -\frac{K_{s}}{\pi} \int_{0}^{\infty} h\left(\beta, y_{s}\right) g(\beta, y) \cos \left[\beta K_{s}\left(x-x_{s}\right)\right] d \beta \\
= & -\frac{K_{s}}{\pi}\left\{\int_{0}^{\beta_{r}-\Delta} h\left(\beta, y_{s}\right) g(\beta, y) \cos \left[\beta K_{s}\left(x-x_{s}\right)\right] d \beta\right. \\
& \left.+\int_{\beta_{r}+\Delta}^{\beta_{\max }} h\left(\beta, y_{s}\right) g(\beta, y) \cos \left[\beta K_{s}\left(x-x_{s}\right)\right] d \beta\right\} \\
& -\frac{i K_{s}}{R} h\left(\beta_{r}, y_{s}\right) \hat{g}\left(\beta_{r}, y\right) \cos \left[\beta_{r} K_{s}\left(x-x_{s}\right)\right] \\
& -\frac{K_{s}}{\pi R}\left\{h\left(\beta_{r}+\Delta, y_{s}\right) \hat{g}\left(\beta_{r}+\Delta, y\right) \cos \left[\left(\beta_{r}+\Delta\right) K_{s}\left(x-x_{s}\right)\right]\right\} \\
& \left.+\frac{K_{s}}{\pi R}\left\{h\left(\beta_{r}-\Delta, y_{s}\right) \hat{g}\left(\beta_{r}-\Delta, y\right) \cos \left[\left(\beta_{r}-\Delta\right) K_{s}\left(x-x_{s}\right)\right]\right\}\right)
\end{aligned}
$$

With antisymmetric transfer function $g(\beta, y)$

$$
\begin{aligned}
g^{B}(x, y)= & -\frac{i K_{s}}{\pi} \int_{0}^{\infty} h\left(\beta, y_{s}\right) g(\beta, y) \sin \left[\beta K_{s}\left(x-x_{s}\right)\right] d \beta \\
= & -\frac{i K_{s}}{\pi}\left\{\int_{0}^{\beta_{r}-\Delta} h\left(\beta, y_{s}\right) g(\beta, y) \sin \left[\beta K_{s}\left(x-x_{s}\right)\right] d \beta w\right\} \\
& \left.+\int_{\beta_{r}+\Delta}^{\beta_{\max }} h\left(\beta, y_{s}\right) g(\beta, y) \sin \left[\beta K_{s}\left(x-x_{s}\right)\right] d \beta\right\} \\
& +\frac{K_{s}}{R} h\left(\beta_{r}, y_{s}\right) \hat{g}\left(\beta_{r}, y\right) \sin \left[\beta_{r} K_{s}\left(x-x_{s}\right)\right] \\
& -\frac{i K_{s}}{\pi R}\left\{h\left(\beta_{r}+\Delta, y_{s}\right) \hat{g}\left(\beta_{r}+\Delta, y\right) \sin \left[\left(\beta_{r}+\Delta\right) K_{s}\left(x-x_{s}\right)\right]\right\} \\
& \left.+\frac{i K_{s}}{\pi R}\left\{h\left(\beta_{r}-\Delta, y_{s}\right) \hat{g}\left(\beta_{r}-\Delta, y\right) \sin \left[\left(\beta_{r}-\Delta\right) K_{s}\left(x-x_{s}\right)\right]\right\}\right)
\end{aligned}
$$

where

$$
R=4\left[2 \beta_{r}\left(2 \beta_{r}{ }^{2}-1\right)-\frac{4 \beta_{r}{ }^{5}-3 \beta_{r}{ }^{3}\left(1+\chi^{2}\right)+2 \beta_{r} \chi^{2}}{\sqrt{\left({\beta_{r}}^{2}-1\right)} \sqrt{\left(\beta_{r}{ }^{2}-\chi^{2}\right)}}\right]
$$




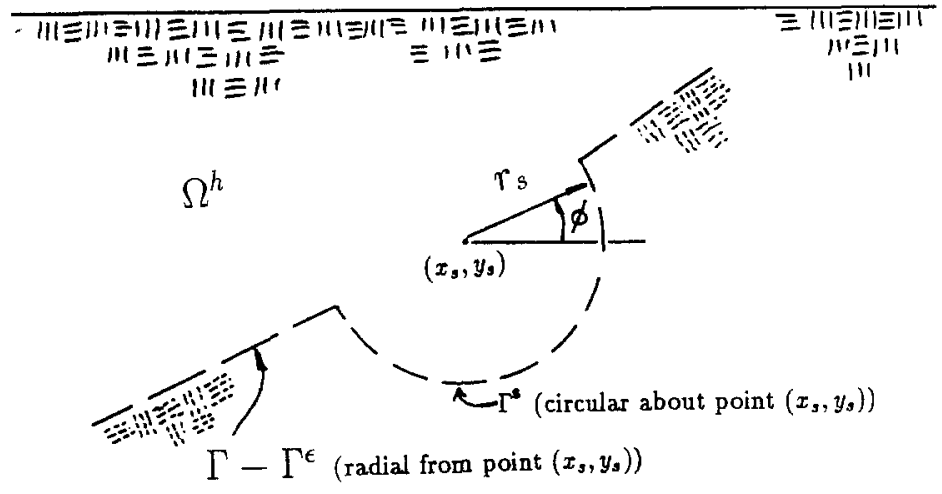

FIG. A3.

APPENDIX 3. Singularities IN SOlUtions For Line LOADS

Notes: $s t$ ( ) denotes singular term in response quantity. \pm means + when material is on the right when facing in the positive radial direction along $\Gamma-\Gamma^{\epsilon}$, and - when material is on the left. Refer $[2,9]$.

Antiplane shear: $\delta_{z}\left(x-x_{s}, y-y_{s}\right)$

$$
\begin{gathered}
s t\left(u_{z}^{*}\right)=-\frac{1}{4 \mu} \ln \left(r_{s}\right) \\
s t\left(T_{z}^{*}\right)=0 \quad \text { on } \Gamma-\Gamma^{\epsilon} \\
s t\left(T_{z}^{*}\right)=+\frac{1}{2 \pi r_{s}} \quad \text { on } \Gamma^{s}
\end{gathered}
$$

Plane strain: buried line load $\delta_{x}\left(x-x_{s}, y-y_{s}\right)$

$$
\begin{gathered}
s t\left(u_{x}^{*}\right)=-\frac{\left(1+\kappa^{2}\right)}{4 \pi C_{p}^{2} \rho} \ln \left(r_{s}\right) \\
s t\left(u_{y}^{*}\right)=0 \\
s t\left(T_{x}^{*}\right)=0 \quad \text { on } \Gamma-\Gamma^{\epsilon} \\
s t\left(T_{y}^{*}\right)= \pm \frac{-1}{2 \pi \kappa^{2} r_{s}} \quad \text { on } \Gamma-\Gamma^{\epsilon} \\
s t\left(T_{x}^{*}\right)=-\frac{C_{1}}{r_{s}}\left[C_{2}+2 \cos ^{2} \phi\right] \quad \text { on } \Gamma^{s} \\
s t\left(T_{y}^{*}\right)=-2 \frac{C_{1}}{r_{s}} \cos \phi \sin \phi \quad \text { on } \Gamma^{s}
\end{gathered}
$$

Plane strain: buried line load $\delta_{y}\left(x-x_{s}, y-y_{s}\right)$

$$
\operatorname{st}\left(u_{x}^{*}\right)=0
$$




$$
\begin{gathered}
\operatorname{st}\left(u_{y}^{*}\right)=-\frac{\left(1+\kappa^{2}\right)}{4 \pi C_{p}{ }^{2} \rho} \ln \left(r_{s}\right) \\
\operatorname{st}\left(T_{x}^{*}\right)= \pm \frac{1}{2 \pi \kappa^{2} r_{s}} \quad \text { on } \Gamma-\Gamma^{\epsilon} \\
s t\left(T_{y}^{*}\right)=0 \quad \text { on } \Gamma-\Gamma^{\epsilon} \\
\operatorname{st}\left(T_{x}^{*}\right)=-2 \frac{C_{1}}{r_{s}} \cos \phi \sin \phi \quad \text { on } \Gamma^{s} \\
\operatorname{st}\left(T_{y}^{*}\right)=-\frac{C_{1}}{r_{s}}\left[C_{2}+2 \sin ^{2} \phi\right] \quad \text { on } \Gamma^{s}
\end{gathered}
$$

Plane strain: line load $\delta_{x}\left(x-x_{s}, y-y_{s}\right)$ at horizontal surface of half space

$$
\begin{gathered}
s t\left(u_{x}^{*}\right)=-\frac{1-\nu}{\pi \mu} \ln \left(r_{s}\right) \\
s t\left(u_{y}^{*}\right)=0 \\
s t\left(T_{x}^{*}\right)=0 \quad \text { on } \Gamma-\Gamma^{\epsilon} \\
s t\left(T_{y}^{*}\right)=0 \quad \text { on } \Gamma-\Gamma^{\epsilon} \\
\operatorname{st}\left(T_{x}^{*}\right)=\frac{2}{\pi r_{s}} \cos ^{2} \phi \quad \text { on } \Gamma^{s} \\
\operatorname{st}\left(T_{y}^{*}\right)=\frac{2}{\pi r_{s}} \sin \phi \cos \phi \quad \text { on } \Gamma^{s}
\end{gathered}
$$

Plane strain: line load $\delta_{y}\left(x-x_{s}, y-y_{s}\right)$ at horizontal surface of half-space

$$
\begin{gathered}
s t\left(u_{x}^{*}\right)=0 \\
s t\left(u_{y}^{*}\right)=-\frac{1-\nu}{\pi \mu} \ln \left(r_{s}\right) \\
s t\left(T_{x}^{*}\right)=0 \quad \text { on } \Gamma-\Gamma^{\epsilon} \\
s t\left(T_{y}^{*}\right)=0 \quad \text { on } \Gamma-\Gamma^{\epsilon} \\
\operatorname{st}\left(T_{x}^{*}\right)=\frac{2}{\pi r_{s}} \sin \phi \cos \phi \quad \text { on } \Gamma^{s} \\
\operatorname{st}\left(T_{y}^{*}\right)=\frac{2}{\pi r_{s}} \sin ^{2} \phi \quad \text { on } \Gamma^{s}
\end{gathered}
$$

where $\kappa=C_{p} / C_{s}, C_{1}=1 /(4 \pi(1-\nu))$ and $C_{2}=1-2 \nu$. 


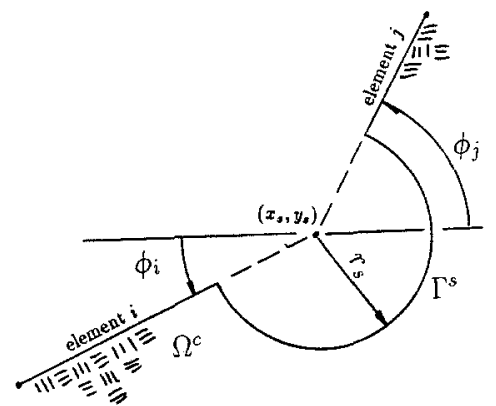

FIG. A4.

APPENDIX 4. INTEgRals OVER $\Gamma^{S}$ OF Line LOAD TRACTIONS Antiplane shear: $\delta_{z}\left(x-x_{s}, y-y_{s}\right)$

$$
\lim _{r_{s} \rightarrow 0} \int_{\Gamma^{s}} T_{z}^{*} d \Gamma^{s}=0.5+\frac{\left(\phi_{j}-\phi_{i}\right)}{2 \pi}
$$

Plane strain: buried line load $\delta_{x}\left(x-x_{s}, y-y_{s}\right)$

$$
\begin{gathered}
\lim _{r_{s} \rightarrow 0} \int_{\Gamma^{s}} T_{x}^{*} d \Gamma^{s}=1.0+C_{1}\left[\left(C_{2}+1\right)\left(\pi+\phi_{i}-\phi_{j}\right)+\frac{1}{2}\left(\sin \left(2 \phi_{i}\right)-\sin \left(2 \phi_{j}\right)\right)\right] \\
\lim _{r_{s} \rightarrow 0} \int_{\Gamma^{s}} T_{y}^{*} d \Gamma^{s}=-C_{1}\left(\sin ^{2} \phi_{j}-\sin ^{2} \phi_{i}\right)
\end{gathered}
$$

Plane strain: buried line load $\delta_{y}\left(x-x_{s}, y-y_{s}\right)$

$$
\begin{gathered}
\lim _{r_{s} \rightarrow 0} \int_{\Gamma^{s}} T_{x}^{*} d \Gamma^{s}=-C_{1}\left(\sin ^{2} \phi_{j}-\sin ^{2} \phi_{i}\right) \\
\lim _{r_{s} \rightarrow 0} \int_{\Gamma^{s}} T_{y}^{*} d \Gamma^{s}=1.0+C_{1}\left[\left(C_{2}+1\right)\left(\pi+\phi_{i}-\phi_{j}\right)-\frac{1}{2}\left(\sin \left(2 \phi_{i}\right)-\sin \left(2 \phi_{j}\right)\right)\right]
\end{gathered}
$$

Plane strain: line load $\delta_{x}\left(x-x_{s}, y-y_{s}\right)$ at top of canyon

$$
\begin{aligned}
\lim _{r_{s} \rightarrow 0} \int_{\Gamma^{s}} T_{x}^{*} d \Gamma^{s} & =\frac{1}{\pi}\left[\left(\pi+\phi_{1}\right)+\frac{1}{2} \sin \left(2 \phi_{1}\right)\right] \quad \text { at node } 1 \\
=\frac{1}{\pi}\left(\pi-\phi_{n-1}-\frac{1}{2} \sin \left(2 \phi_{n-1}\right)\right) \quad \text { at node } n-1 & (\mathrm{D}) \quad \text { at node } 1 \\
\lim _{r_{s} \rightarrow 0} \int_{\Gamma^{s}} T_{y}^{*} d \Gamma^{s} & =\frac{1}{\pi} \sin ^{2} \phi_{1} \quad \text { at node } n-1 \\
& =-\frac{1}{\pi} \sin ^{2} \phi_{n-1} \quad
\end{aligned}
$$


Plane strain: line load $\delta_{y}\left(x-x_{s}, y-y_{s}\right)$ at top of canyon

$$
\begin{gathered}
\lim _{r_{s} \rightarrow 0} \int_{\Gamma^{s}} T_{x}^{*} d \Gamma^{s}=\frac{1}{\pi} \sin ^{2} \phi_{1} \quad \text { at node } 1 \\
=-\frac{1}{\pi} \sin ^{2} \phi_{n-1} \quad \text { at node } n-1 \\
\lim _{r_{s} \rightarrow 0} \int_{\Gamma^{s}} T_{y}^{*} d \Gamma^{s}=\frac{1}{\pi}\left[\left(\pi+\phi_{1}\right)-\frac{1}{2} \sin \left(2 \phi_{1}\right)\right] \quad \text { at node } 1 \\
=\frac{1}{\pi}\left(\pi-\phi_{n-1}-\frac{1}{2} \sin \left(2 \phi_{n-1}\right)\right) \quad \text { at node } n-1
\end{gathered}
$$

where $C_{1}=1 /(4 \pi(1-\nu))$ and $C_{2}=1-2 \nu$.

Associate Professor of Civil Engineering

\section{SCHOOL OF ENGINEERING}

GONZOGA UNIVERSITY

SPOKANE, WASHINGTON 99258 (P.S.N.)

Manuscript received 20 July 1993
Associate Professor

Department of Civil Engineering

California Institute of Technology

Pasadena, CAlifornia 91125 (J.F.H.) 